\title{
Las narrativas de los planes gubernamentales como una estrategia para la imposición de un nuevo ciclo de explotación en la región amazónica: el caso de las hidroeléctricas en Brasil
}

As narrativas dos planos governamentais como estratégia para a imposição de um novo ciclo de exploração na região amazônica: o caso das hidrelétricas no Brasil

The narratives of government plans as a strategy for the imposition of a new exploration cycle in the amazon region: the case of hydroelectric plants in Brazil

AUTORES

Pedro Abib

Hecktheuer

pedro@fcr.edu.br

Maria Cláudia da Silva Antunes de Souza*

mclaudia@univali.br

* Profesor de Derecho Constitucional en la Faculdade de Direito da Católica de Rondônia (FCR, Brasil).

** Profesora del programa de posgrado en Ciencia Jurídica de la Universidade do Vale do Itajaí (Univali, Brasil).

\section{RESUMEN:}

Esta investigación tiene como objetivo evaluar si las narrativas de que la implementación de Centrales Hidroeléctricas son el mejor camino para el desarrollo y una fuente de energía limpia y sostenible representa una estrategia para la imposición de un nuevo ciclo de explotación de la región amazónica, caracterizado por imponer altos costos socioambientales para la región. Con este fin, se discute la resignificación otorgada a los ríos y a la región amazónica en los planes del gobierno brasileño, especialmente a partir de la Operación Amazonia, desde la década de 1960. La Amazonia recibió especial atención del gobierno brasileño en la expansión de las fuentes hidroeléctricas en el sector energético brasileño, sin que hubiera ninguna preocupación con relación a los impactos socioambientales de tales proyectos. Las narrativas y documentos oficiales, sin demostrar veracidad, impusieron la expansión hidroeléctrica como la única forma para el desarrollo del país, lo que hizo posible la construcción de varias centrales hidroeléctricas en la Amazonia, con resultados trágicos para los pueblos y comunidades tradicionales. Para obtener tales consideraciones, se realizó una investigación bibliográfica y documental.

\section{RESUMO:}

O presente artigo tem como objetivo avaliar se as narrativas de que a implementação de Usinas Hidrelétricas são o melhor caminho para o desenvolvimento e uma fonte de energia limpa e sustentável representa uma estratégia para a imposição de um novo ciclo de exploração da região amazónica, caracterizado por impor elevados custos socioambientais à região. Para tanto, discute-se a ressignificação que se deu aos rios e a região amazónica nos planos do governo brasileiro, em especial a partir da Operação Amazônia, a partir da década de 1960. A Amazônia ganhou especial atenção do governo para a expansão do setor energético brasileiro, sem nenhum tipo de preocupação com os impactos socioambientais que tais empreendimentos causariam. As narrativas e documentos oficiais, sem demonstrar a veracidade, impuseram a expansão hidrelétrica como o único caminho para o desenvolvimento do país, o que viabilizou tais empreendimentos na Amazônia, mesmo com trágicos resultados para os povos e comunidades tradicionais. Para que fosse possível obter tais considerações, empreendeu-se uma pesquisa bibliográfica e documental. 


\section{ABSTRACT:}

This article aims to assess whether the narratives that the implementation of Hydroelectric Plants are the best path for national development and that they are a source of clean and sustainable energy represents a strategy for the imposition of a new cycle of exploration in the Amazon region, characterized by imposing high socio-environmental costs on the region. To this end, it is discussed the resignification that was given to the rivers and to the Amazon region in the plans of the Brazilian government, especially after Amazon Operation, from the 1960s. The Amazon gained special attention from the government for the expansion of the energy sector, without any concern with the socio-environmental impacts that such projects would cause. The official narratives and documents, without demonstrating their veracity, imposed hydroelectric expansion as the only way for the development of the country, which made such ventures possible in the Amazon, even with tragic results for traditional peoples and communities. In order to obtain such considerations, a bibliographical and documentary research was undertaken. 


\section{Introducción}

Esta investigación tiene como objetivo evaluar si las narrativas de que las represas hidroeléctricas son una fuente de energía limpia y sostenible representan una estrategia para la imposición de un nuevo ciclo de explotación de la región amazónica, con beneficios exógenos y pérdidas socioambientales locales.

La región pasó por varios ciclos de ocupación y explotación, siempre con imposiciones exógenas y en detrimento de sus pueblos y comunidades tradicionales, ya sea en el período colonial, con las misiones militares e incursiones, en la política de Pombal o en los ciclos del látex. Esta realidad, sin embargo, no parece haber sido superada. El origen del problema amazónico es eminentemente político, en su sentido más amplio. Tal problema invariablemente afecta manera sustancial a los pueblos y comunidades tradicionales y su sostenibilidad.

Con base en eso, el problema de la investigación reside en la siguiente pregunta: ¿son las narrativas encontradas en los planes del gobierno brasileño, que presentan los proyectos hidroeléctricos como una fuente limpia y sostenible, una forma de dirigir el sector energético brasileño a la región amazónica, independientemente de los desafíos socioambientales, o efectivamente corresponde a la realidad de los proyectos implementados en la Amazonia?

Como hipótesis, se tiene la intuición de que las narrativas y discursos gubernamentales son una estrategia para imponer intereses exógenos, una vez que los proyectos hidroeléctricos llevados a cabo en la región amazónica suponen graves daños socioambientales, representando poco o casi ningún beneficio para los pueblos y comunidades tradicionales de la Amazonia.

Para poder resolver el problema, se realizó una investigación bibliográfica y documental. Además, en las diversas fases de la investigación, se utilizaron las técnicas del referente, de la categoría y del concepto operativo (Pasold, 2015).

\section{La resignificación de los ríos y las aguas amazónicas en Brasil}

La percepción de que el agua es inagotable y que es un elemento abundante ejerció una fuerte influencia en la formación socioeconómica brasileña, especialmente en la Amazonia. Como la región amazónica se caracteriza por una infinidad de cuerpos de agua y de las más amplias dimensiones, esta percepción está presente con un cierto sobredimensionamiento tanto en los libros como en los discursos oficiales, informes y planes gubernamentales.

Siempre hubo una fuerte interacción entre los ríos y las sociedades humanas que han vivido en la región durante siglos. El río Amazonas y sus afluentes han sido estratégicos en términos de observación y movimiento de personas y bienes, además de constituir el camino que condujo a la región. Durante mucho tiempo, la historiografía amazónica entendió sus ríos como un lugar de "tráfico de personas, productos, cultura civilizadora o como un lugar de referencia para las poblaciones nativas, pueblos y ciudades, siempre como una ruta acuática" (Batista, 2019, p. 122).

Por otro lado, desde una perspectiva histórico-ambiental, las producciones historiográficas sobre la Amazonia, actualmente, han problematizado las aguas al abordar las dinámicas socioeconómicas, políticas, culturales y ambientales que se llevan a cabo en sus márgenes y en su curso. Las aguas de los ríos amazónicos ahora han ganado una perspectiva comercial. Los sentidos y los significados que les fueron atribuidos por sus poblaciones tradicionales ya no son los mismos.
PALABRAS CLAVE Centrales

hidroeléctricas;

Amazonia; planes

del gobierno;

socioambiental.

PALAVRAS-CHAVE

Hidrelétricas;

Amazônia; planos

do governo;

socioambiental.

KEYWORDS

Hydroelectric

plants; Amazon;

government

plans; socio-

environmental.

Recibido:

20/05/2020

Aceptado:

20/09/2020 
Los procesos de ocupación y reocupación de la Amazonia tienen una base sólida en sus ríos. En el más reciente, las aguas amazónicas fueron "resignificadas en kilovatios y mercancías, poniendo en un segundo plano las relaciones mantenidas por las comunidades/pueblos con los ríos" (Batista, 2019, p. 117). Esto se debió a los grandes proyectos minero-metalúrgicos e hidroeléctricos que se han desarrollado desde hace algunas décadas, que han dado centralidad a los ríos de la región, sirviendo, ahora, como una fuerza impulsora para las operaciones industriales y urbanas en el país.

Esta perspectiva de convertir las aguas de los ríos en bienes y capital ha estado presente en el imaginario de los gobiernos brasileños al formular sus planes de desarrollo a lo largo del siglo XX. Entre los años 1930 y 1960, la energía hidroeléctrica brasileña pasó de 615 a 3,642.0 megavatios (MW), todavía restringida a la explotación energética de los ríos que estaban cerca de los grandes centros industriales del país. Con el agotamiento de estas fuentes cercanas, más tarde se comenzaron a buscar otras cuencas hidrográficas (Batista, 2019, p. 126). En particular, la matriz energética brasileña presentó una fuerte inclinación hacia la energía hidroeléctrica a partir de principios de la segunda mitad del siglo XX, cuando comenzó la búsqueda del potencial de los recursos naturales y la posibilidad de su explotación, para lo que se realizaron levantamientos técnicos y planes de desarrollo para el país.

Naturalmente, los documentos gubernamentales pasaron a señalar el potencial energético que tenían los ríos en la región amazónica, después de mapear los lugares que podrían ofrecer el mejor costo-beneficio para tales implementaciones. El gran potencial de la región se debe a las enormes cantidades de agua que la atraviesan y a las importantes caídas topográficas que tienen los afluentes del río Amazonas (Fearnside, 2015, p. 12).

El primer hito legal de este cambio de orientación hacia el potencial energético de los ríos amazónicos tuvo lugar con el Plan de Valorización Económica de la Amazonia, previsto en la Constitución Federal de 1946. Este plan definió como su objetivo el de "establecer una política energética en la región bajo bases económicas" (Brasil, 1953), contemplando la electrificación de los principales centros de producción e industria.

Esa idea se empezó a desarrollar con lo que se denominó "Operación Amazonia" (Brasil, 1968), en la segunda mitad de la década de 1960. Esta operación suponía un plan de desarrollo regional, que buscaba reformular la política económica de la Amazonia, a través de polos de desarrollo que, al privilegiar los incentivos financieros y fiscales para ciertos sectores, buscaba atraer a la población y "ocupar espacios vacíos" (Nahum, 2011, p. 18).

Para hacer esto posible, se realizaron inversiones en infraestructura básica, como carreteras y electricidad. Por lo tanto, el nuevo conjunto de políticas federales para el desarrollo de la Amazonia tenía, entre sus objetivos, la expansión sistemática del sistema de generación de energía existente en el país. Además de todas las estructuras institucionales y organizativas desarrolladas para llevar a cabo estos planes de desarrollo para la Amazonia, en 1968 se creó el Comité Coordinador de Estudios Energéticos de la Amazonia (ENERAM), que estaba vinculado al Ministerio de Minas y Energía (MME) (Brasil, 1968).

Su creación tuvo como objetivo, entre otros, llevar a cabo la supervisión de estudios relacionados con el aprovechamiento del potencial "hidroeléctrico para suministrar los sistemas eléctricos que ya existen o que serían implementados en áreas prioritarias y polos de desarrollo creados en la Amazonia por el Gobierno Federal" (Brasil, 1968b) ${ }^{1}$.

Algunos años después, con la previsión de los polos de desarrollo de la Amazonia (Polo Amazonia), en el Plan de Desarrollo del gobierno de Geisel (Brasil, 1976), se comenzó a privilegiar proyectos a gran escala, intensivos en capital y dirigidos, entre otros sectores, a la producción de energía.

A pesar de que el potencial de producción de energía en la región amazónica se había previsto en los planteamientos de Primer Plan Quinquenal de la Superintendencia del Plan de Valorización Económica de la Amazonia (SPVEA), en 1955, fue solo a partir de la década de 1970 cuando las "condiciones económicas para su la utilización efectiva se materializaron" (Batista, 2019, p. 127). 
- LAS NARRATIVAS DE LOS PLANES GUBERNAMENTALES COMO UNA ESTRATEGIA PARA LA IMPOSICIÓN DE UN NUEVO CICLO DE EXPLOTACIÓN EN LA REGIÓN AMAZÓNICA: EL CASO DE LAS HIDROELÉCTRICAS EN BRASIL PEDRO ABIB HECKTHEUER E MARIA CLÁUDIA DA SILVA ANTUNES DE SOUZA

Dada la importancia del sector, se creó la empresa Centrais Elétricas do Norte do Brasil (Eletronorte)2, una subsidiaria de Centrais Elétricas do Brasil (Eletrobras), que se estableció oficialmente en 1973 (Brasil, $1961 ; 1972 ; 173)$, lo que representa un hito importante para el gobierno federal en la gestión del agua como recurso hídrico en la Amazonia. Su objetivo era realizar estudios, proyectos, construcción y operación de sistemas hidroeléctricos y de transmisión, coordinando programas en el sector eléctrico en la Amazonia (Batista, 2019, p. 127).

Los planes iniciales para el sector energético en la región amazónica comprendían docenas de represas grandes y pequeñas, aprovechando el potencial para generar energía de los afluentes del río Amazonas. La construcción de 79 centrales hidroeléctricas en la Amazonia brasileña (Mapa 1) estaba prevista, a través del Plan Nacional de Electricidad, en 1987 (Brasil, 1987). En ese momento, había un potencial estimado de alrededor de 100 mil MW de generación de energía en la Amazonia (Junk \& Mello, 1987, p. 126).

En el plan gubernamental (Brasil, 1987), el Amazonas representaba, efectivamente, un espacio privilegiado para la expansión de la matriz eléctrica del país. Para el gobierno federal, en este plan, la mayor parte del potencial hidroeléctrico no utilizado del país se encontraba en la Amazonia. Enfatizaba, aún, que la generación de energía en la Amazonia podría transportarse a costes competitivos a las regiones noreste y sureste. También se predijo que la fuente básica para la generación de electricidad en el país seguiría siendo hidroeléctrica hasta 2010. En ese momento, la fuente hidráulica ya representaba el $90 \%$ de la potencia instalada total del país (Brasil, 1987) .

Las dificultades financieras enfrentadas después del período de los gobiernos militares obligaron al país a posponer repetidamente los planes para construir represas, aunque no se pasó por alto la serie planificada. La inundación que generarían todas estas construcciones planificadas alcanzaría los 10 millones de hectáreas, aproximadamente el $2 \%$ de la región de la Amazonia legal, o el 3\% de toda la porción brasileña de la selva amazónica (Fearnside, 2015, p. 13).

El crecimiento de la matriz hidroeléctrica planificada para la región amazónica continuó presente en los planes de las décadas siguientes. Esto se debe a que las acciones propuestas por los programas gubernamentales de Fernando Henrique Cardoso, Luiz Inácio Lula da Silva, Dilma Rousseff y Jair Bolsonaro, han mantenido la misma postura en el sentido de implementar megaproyectos hidroeléctricos en la región.

Desde el Plan 2010, se han presentado un conjunto de Planes Plurianuales (PPA) - que prevén inversiones del gobierno federal por períodos de cuatro años - importantes para el sector energético brasileño, entre los cuales están: Brasil em Ação (Brasil, 1996), Avança Brasil (Brasil, 2000), PPA (Brasil, 2003), Programa de Aceleração do Crescimento (PAC) (Brasil, 2008) y Programa de Aceleração do Crescimento - 2 (PAC-2) (Brasil, 2012). Un aspecto muy relevante de estos planes, y que es importante para los propósitos de este artículo, es el de las grandes obras hidroeléctricas planificadas ${ }^{5}$, muchas de ellas ya ejecutadas, para la Amazonia.

Muchos pronósticos de los primeros planes hidroeléctricos para la Amazonia no se materializaron por varias razones, en particular debido a limitaciones/resistencia económica o

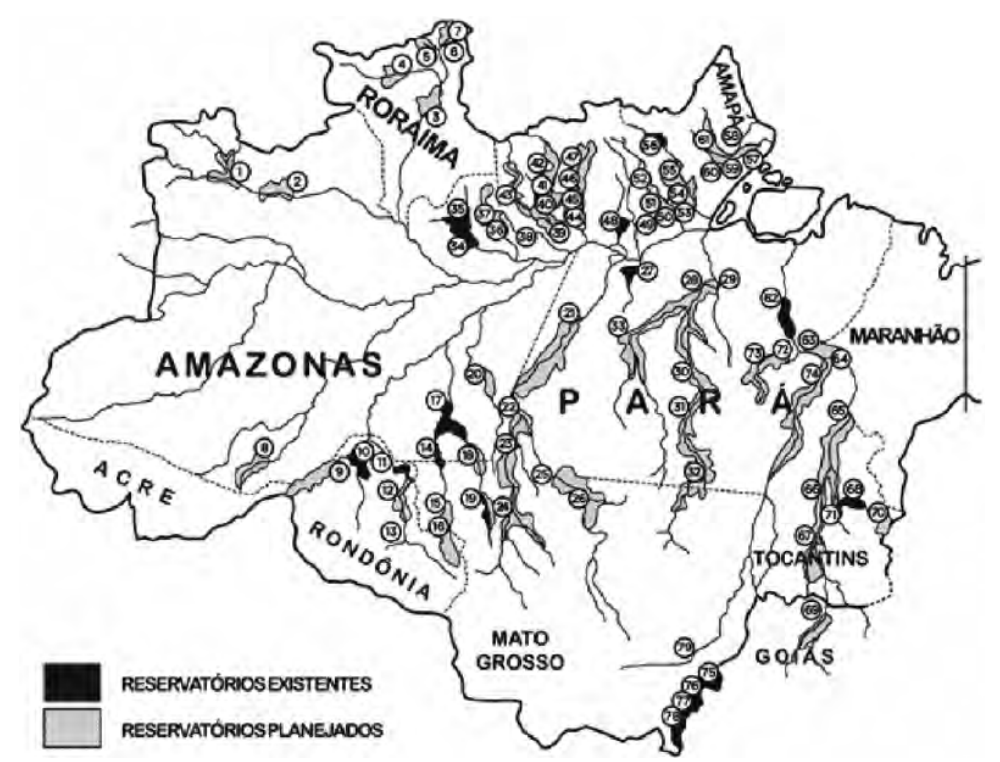

Mapa 1. Mapa del aprovechamiento hidroeléctrico en las cuencas del Amazonas y Tocantins/Araguaia en la década de $1980^{3}$. Fuente: Fearnside, 2015, p. 13. 
- LAS NARRATIVAS DE LOS PLANES GUBERNAMENTALES COMO UNA ESTRATEGIA PARA LA IMPOSICIÓN DE UN NUEVO CICLO DE EXPLOTACIÓN EN LA REGIÓN AMAZÓNICA: EL CASO DE LAS HIDROELÉCTRICAS EN BRASIL • PEDRO ABIB HECKTHEUER E MARIA CLÁUDIA DA SILVA ANTUNES DE SOUZA

política. Durante mucho tiempo, tales proyectos permanecieron latentes, habiendo resurgido recientemente, en función de una situación política más favorable (Fearnside \& Laurence, 2012, pp. 91-92).

Estas estrategias de desaparición/reaparición de proyectos en planes oficiales son características de este sector, y pueden confirmarse observando los diferentes planes gubernamentales y planes decenales de expansión de energía eléctrica ${ }^{6}$, como son ejemplo los complejos Belo Monte y Río Madeira.

Las plantas hidroeléctricas representan un nuevo ciclo de cambios profundos en la región amazónica. Las consecuencias de la construcción de represas hidroeléctricas en la Amazonia son devastadoras. Los impactos socioambientales causados son demasiado significativos. Sin embargo, estos - los impactos socioambientales - no eran una preocupación del gobierno brasileño (Carvalho, 2001, p. 18). En el período en que comenzó la política energética para la Amazonia, no había ningún control con respecto a los impactos que tales proyectos generarían, lo importante era, exclusivamente, la producción de energía y el supuesto desarrollo nacional.

Los primeros grandes proyectos hidroeléctricos en la región fueron: Coaracy-Nunes, Curuá-Una, Tucuruí, Balbina, Mansa y Samuel. Sin excepción, son proyectos cuestionados por la comunidad nacional e internacional, con respecto a sus impactos socioambientales, hasta el día de hoy (Garcia, 2004). Los impactos socioambientales, se reitera, no eran parte de las preocupaciones del gobierno.

Cabe señalar que, en esta fase inicial, existía una estrecha e íntima relación entre los proyectos hidroeléctricos y el desarrollo del sector minero en la Amazonia. La Hidroeléctrica Coaracy Nunes, por ejemplo, mantuvo una asociación directa con la explotación de manganeso en el territorio de Amapá; La de Cachoeira Porteira estaba relacionada con la explotación de la bauxita; La de Samuel, en Rondônia, estuvo motivada por la explotación de casiterita en la región, entre otros (Batista, 2019, p. 127). Posteriormente, esta relación dejó de existir.

Como se mencionó, la expansión energética en la Amazonia fue perenne en los planes gubernamentales, independientemente del sesgo político-ideológico de cada gobierno. No al ritmo o en la cantidad que se planeaban, pero los proyectos hidroeléctricos han sido aprobados, ejecutados e implementados por todos los gobiernos en las últimas décadas. Ya son más de 20 plantas hidroeléctricas en activo en la región Amazónica (Tabla 1).

La realidad amazónica comenzó a reconfigurarse con la presencia de estas innumerables centrales hidroeléctricas (Mapa 2), y es posible confirmar, al observar la tabla, la opción hecha por el gobierno brasileño en la "Operación Amazonia", en los años 60.

Además de los proyectos hidroeléctricos ya implementados, con respecto a la expansión energética en la región, los planes del gobierno prevén una serie de nuevos proyectos hidroeléctricos. Al realizar el análisis de los últimos 12 planes decenales de expansión energética de Brasil se puede observar una larga lista de represas hidroeléctricas a implementar en la Amazonia. Hay más de 43 indicaciones de plantas hidroeléctricas para la región amazónica por parte del gobierno federal (Tabla 2). Muchas de ellas, es cierto, ya no están presentes en los planes más recientes.

Además de los diversos proyectos indicados en los planes analizados (Tabla 2), Fearnside (2015, p. 15) afirma que hay varias represas no inventariadas, "como las 62 represas adicionales

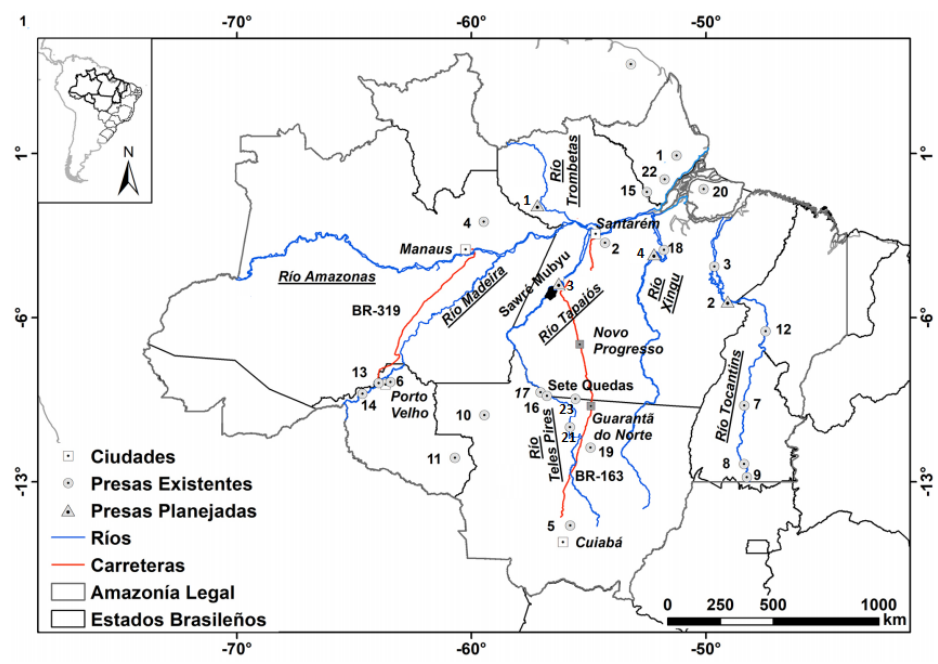

Mapa 2. Represas hidroeléctricas ${ }^{8}$ en la región de la Amazonia legal de Brasil. Fuente: Adaptada de Fearnside (2019, p. 125). 
- LAS NARRATIVAS DE LOS PLANES GUBERNAMENTALES COMO UNA ESTRATEGIA PARA LA IMPOSICIÓN DE UN NUEVO CICLO DE EXPLOTACIÓN EN LA REGIÓN AMAZÓNICA: EL CASO DE LAS HIDROELÉCTRICAS EN BRASIL PEDRO ABIB HECKTHEUER E MARIA CLÁUDIA DA SILVA ANTUNES DE SOUZA

Tabla 1. Plantas hidroeléctricas en la Amazonia Legal brasileña con más de 30 MW de capacidad instalada (1975-2019).

\begin{tabular}{|c|c|c|c|c|}
\hline AÑO & NOMBRE & ESTADO & Río & CAPACIDAD (MW) \\
\hline 1975 & Coaracy-Nunes & Amapá & Araguari & 298 \\
\hline 1977 & Curuá-Una & Pará & Curuá-Una & 100 \\
\hline 1984 & Tucuruí & Pará & Tocantins & 8370 \\
\hline 1987 & Balbina & Amazonas & Uatumá & 250 \\
\hline 1987 & Manso & Mato Grosso & Manso & 212 \\
\hline 1988 & Samuel & Rondônia & Jamari & 210 \\
\hline 1999 & Lajeado & Tocantins & Tocantins & 800 \\
\hline 2006 & Peixe Angical & Tocantins & Tocantins & 452 \\
\hline 2009 & São Salvador & Tocantins & Tocantins & 243.2 \\
\hline 2011 & Dardanelos & Mato Grosso & Aripuanã & 261 \\
\hline 2011 & Santo Antônio & Rondônia & Madeira & 3568 \\
\hline 2011 & Rondon II & Rondônia & Comemoração & 73.5 \\
\hline 2012 & Estreito (Tocantins) & Maranhão/Tocantins & Tocantins & 1087 \\
\hline 2013 & Jirau & Rondônia & Madeira & 3750 \\
\hline 2014 & Santo Antônio do Jari & Pará/Amapá & Jari & 373 \\
\hline 2014 & Sinop & Mato Grosso & Teles Pires & 401.88 \\
\hline 2014 & São Manoel & Pará/Mato Grosso & Teles Pires & 700 \\
\hline 2014 & Ferreira Gomes & Amapá & Araguari & 252 \\
\hline 2015 & Teles Pires & Pará/Mato Grosso & Teles Pires & 1820 \\
\hline 2016 & Complexo Apaiacás & Mato Grosso & Teles Pires & 102 \\
\hline 2016 & Cachoeira Caldeirão & Amapá & Araguari & 219 \\
\hline 2018 & Colider & Mato Grosso & Teles Pires & 300 \\
\hline 2019 & Belo Monte & Pará & Xingu & 11.233 .1 \\
\hline
\end{tabular}

Fuente: Elaboración propia a partir de varias fuentes ${ }^{7}$.

que se incluyeron en el Plan 2010". Muchos de los que se han enumerado en esta tabla ya se encuentran en etapas avanzadas de estudio e implementación.

Un aspecto muy importante en el proceso de toma de decisiones para comenzar nuevos proyectos son los impactos sociales y ambientales de estas represas en la región. No se puede subestimar tales impactos $y$, por otro lado, tampoco se puede sobreestimar o considerar solo los beneficios, como viene siendo la práctica tanto en las narrativas gubernamentales como de las empresas constructoras. Las poblaciones locales a menudo reciben los principales impactos, mientras que las recompensas benefician en gran medida a centros urbanos distantes (Fearnside, 2015, p. 14).

No hay forma de saber el porqué, pero el hecho es que, para complicar aún más la situación, la franja donde se encuentran los lugares más favorables para la construcción de represas hidroeléctricas también es la que tiene la mayor concentración de pueblos indígenas en la Amazonia (Fearnside, 2015, p. 13). Es importante, con el 
- LAS NARRATIVAS DE LOS PLANES GUBERNAMENTALES COMO UNA ESTRATEGIA PARA LA IMPOSICIÓN DE UN NUEVO CICLO DE EXPLOTACIÓN EN LA REGIÓN AMAZÓNICA: EL CASO DE LAS HIDROELÉCTRICAS EN BRASIL PEDRO ABIB HECKTHEUER E MARIA CLÁUDIA DA SILVA ANTUNES DE SOUZA

Tabla 2. Proyectos hidroeléctricos para la Amazonia Legal previstos en los Planes Gubernamentales ${ }^{9}$

\begin{tabular}{|c|c|c|c|c|}
\hline $\begin{array}{l}\text { PREVISIÓN DE } \\
\text { ENTRADA EN } \\
\text { OPERACIÓN } \\
\end{array}$ & PROYECTO & $\begin{array}{l}\text { CAPACIDAD } \\
\text { (MW) }\end{array}$ & & FUENTE \\
\hline \multicolumn{5}{|l|}{ AÑO } \\
\hline Indefinido & UHE Babaquara (Altamira) & & 6140 & Fearnside, 2006 \\
\hline Indefinido & UHE Santa Isabel & & 1080 & Fearnside, 2015 \\
\hline Indefinido & UHE Paiaguá & & 35.2 & Brasil, 2006 \\
\hline Indefinido & UHE Taricoejo & & 76 & Brasil, 2006 \\
\hline Indefinido & UHE Tupirantins & & 620 & Brasil, 2006 \\
\hline Indefinido & UHE Uruçui & & 164 & Brasil, 2006 \\
\hline Indefinido & UHE Cachoeira do Caracol & & 32,6 & Brasil, 2007 \\
\hline Indefinido & Cachoeira do Meio & & 44,9 & Brasil, 2007 \\
\hline Indefinido & Cachoeira Fortaleza & & 50,8 & Brasil, 2007 \\
\hline Indefinido & Cachoeira São José & & 36 & Brasil, 2007 \\
\hline Indefinido & UHE Tocantins Renascer & & 480 & Brasil, 2007 \\
\hline Indefinido & UHE Magessi & & 53 & Brasil, 2007 \\
\hline Indefinido & UHE Paraná & & 95 & Brasil, 2008 \\
\hline Indefinido & UHE Pau D'Arco & & 64 & Brasil, 2008 \\
\hline Indefinido & UHE Barra da Palma & & 58 & Brasil, 2008 \\
\hline Indefinido & UHE Berimbau & & 26 & Brasil, 2008 \\
\hline Indefinido & UHE Brejão & & 75 & Brasil, 2008 \\
\hline Indefinido & UHE Cachoeirão & & 64 & Brasil, 2008 \\
\hline Indefinido & UHE Couto Magalhães & & 150 & Brasil, 2008 \\
\hline Indefinido & UHE Ipueiras & & 480 & Brasil, 2008 \\
\hline Indefinido & UHE Jardim de Ouro & & 227 & Brasil, 2008 \\
\hline Indefinido & UHE Juruena & & 46 & Brasil, 2008 \\
\hline Indefinido & UHE Mocotó & & 95 & Brasil, 2008 \\
\hline Indefinido & UHE Jutuarama & & 66 & Brasil, 2008 \\
\hline Indefinido & UHE Novo Acordo & & 160 & Brasil, 2008 \\
\hline 2019 & UHE Cachoeira dos Patos & & 528 & Brasil, 2011 \\
\hline 2019 & UHE Marabá & & 2160 & Brasil, 2013 \\
\hline 2020 & UHE Jamanxim & & 881 & Brasil, 2013 \\
\hline 2020 & UHE Cachoeira do Caí & & 802 & Brasil, 2013 \\
\hline 2020 & UHE Serra Quebrada & & 1328 & Brasil, 2013 \\
\hline 2020 & UHE Agua Limpa & & 380 & Brasil, 2013 \\
\hline
\end{tabular}


- LAS NARRATIVAS DE LOS PLANES GUBERNAMENTALES COMO UNA ESTRATEGIA PARA LA IMPOSICIÓN DE UN NUEVO CICLO DE EXPLOTACIÓN EN LA REGIÓN AMAZÓNICA: EL CASO DE LAS HIDROELÉCTRICAS EN BRASIL PEDRO ABIB HECKTHEUER E MARIA CLÁUDIA DA SILVA ANTUNES DE SOUZA

\begin{tabular}{|llll|}
\hline $\begin{array}{l}\text { PREVISIÓN DE } \\
\text { ENTRADA EN } \\
\text { OPERACIÓN }\end{array}$ & PROYECTO & $\begin{array}{l}\text { CAPACIDAD } \\
\text { (MW) }\end{array}$ & FUENTE \\
\hline AÑO & & & \\
\hline 2022 & UHE Salto Augusto Baixo & 1461 & Brasil, 2013 \\
\hline 2022 & UHE São Simão Alto & 3509 & Brasil, 2013 \\
\hline 2022 & UHE Arraias & 70 & Brasil, 2014 \\
\hline 2022 & UHE Prainha & 796 & Brasil, 2014 \\
\hline 2023 & UHE Paredão A & 199 & Brasil, 2014 \\
\hline 2023 & UHE Torixoréu & 408 & Brasil, 2014 \\
\hline 2021 & UHE São Luiz do Tapajós & 6133 & Brasil, 2015 \\
\hline 2026 & UHE Castanheira & 140 & Brasil, 2020 \\
\hline 2027 & UHE Tabajara & 400 & Brasil, 2020 \\
\hline 2028 & UHE Bem Querer & 650 & Brasil, 2020 \\
\hline DESPUES DE 2029 & UHE Jatobá & 1650 & Brasil, 2020 \\
\hline DESPUES DE 2029 & UHE Paranã & 90 & Brasil, 2020 \\
\hline
\end{tabular}

Fuente: Elaboración propia.

propósito de aclarar este problema, comprender de manera más amplia cuáles son los impactos directos que sufren los pueblos y las comunidades tradicionales en las experiencias hidroeléctricas de la región.

Desde las primeras implementaciones de proyectos hidroeléctricos en la Amazonia, como ya se mencionó, la mayoría de los costos recayeron sobre el medio ambiente, incluyendo los pueblos y las comunidades tradicionales.

Los pueblos y las comunidades tradicionales de la Amazonia interactúan de manera sostenible con la naturaleza, porque es allí donde encuentran su razón de vivir, es allí donde experimentan sus culturas, por lo que todo ello - el entorno natural, los árboles, los ríos, los animales - constituye la vida misma de los pueblos y comunidades tradicionales. Cualquiera que sea el impacto en esta relación, significa dañar irreversiblemente su existencia.

\section{Las narrativas de los planes gubernamentales como estrategia para la expansión del sector energético brasileño: la persistencia de un patrón colonial impuesto a la región}

Las políticas y planes del gobierno brasileño, especialmente aquellos dirigidos a la matriz hidroeléctrica en la Amazonia, se guiaron por la desinformación, o incluso por información incompleta, distorsionada o falsa. Las declaraciones falsas se usan como verdaderas con la intención de engañar al destinatario. En tales proyectos, la práctica y estrategia es la exaltación de los beneficios y la infravaloración de los costos sociales/humanos y ambientales.

Los impactos sociales, sobre los pueblos y comunidades tradicionales, sin embargo, son muchos. Algunos de ellos son: la pérdida de peces y recursos a los que estas personas tenían acceso en los ríos; reasentamientos en realidades completamente diferentes de aquellas en las que tienen sus formas de vida y preservación cultural, en relación con el río y la naturaleza, por lo tanto, generando una pérdida del estilo 
de vida y la forma de subsistencia de estas poblaciones; problemas de salud debido a la proliferación de insectos y metilación del mercurio, entre tantos otros impactos (Fearnside, 2019, p. 56).

Los costos ambientales, asimismo, son enormes. Los impactos en el medio ambiente implican, entre otras cosas, la emisión de gases de efecto invernadero, en particular de dióxido de carbono, que se produce por la descomposición de los árboles muertos por la inundación; por el óxido nitroso y el metano del agua en los depósitos y también en el agua que pasa a través de turbinas y vertederos, lo que representa un importante medio de calentamiento global; además de la pérdida de ecosistemas, fauna y flora (Fearnside, 2019, p. 56).

Como se ha mencionado, la falta de información en los discursos oficiales sobre estas fuentes hidroeléctricas y sus impactos es muy grande. Muy poco o casi nada se dice sobre los gases de efecto invernadero emitidos por las represas, tampoco sobre los desastres sociales y humanos de estos proyectos.

Tales proyectos continúan siendo tratados, en las narrativas y documentos, como una fuente de energía "limpia", y considerados como proyectos de emisiones bajas o insignificantes. Los estudios demuestran que los impactos sobre el calentamiento global son demasiado significativos, hasta el punto de que las emisiones de represas hidroeléctricas equivalen a las emisiones de grandes capitales o incluso de fuentes de energía que utilizan combustibles fósiles. Los impactos son todavía más significativos cuando se comparan con fuentes como la eólica o la solar (Fearnside, 2019, p. 56).

No obstante, los Estudios de Impacto Ambiental ${ }^{10}$ no lo tratan así, tampoco las narrativas gubernamentales. La opinión dominante sigue siendo que las represas hidroeléctricas producen energía limpia, un problema repetido reiteradamente por el gobierno brasileño y las industrias hidroeléctricas y de aluminio.

Dado este escenario, y aunque este no es el objeto de esta investigación, es necesario plantear algunas preguntas. Entre ellas: ¿son las represes hidroeléctricas la “única opción” para el sector energético brasileño? ¿Existen formas y medidas alternativas para aumentar la eficiencia del uso de la electricidad en Brasil? o, aún si ¿existe la posibilidad de simplemente no generar más electricidad en el país?

No se pretende responder a estas preguntas con profundidad, ya que, como se ha dicho, no es el objetivo de este trabajo. Incluso porque estas respuestas no resultan, por favorables que puedan ser para la construcción de plantas hidroeléctricas, esenciales y justificativas para imponer tales costos sociales y humanos a los pueblos y comunidades en la Amazonia. Sin embargo, es importante realizar algunos comentarios sobre tales cuestiones.

La narrativa de los defensores de la construcción de hidroeléctricas es siempre que las represas son el único camino para el desarrollo del país o de la región, o que son la única forma de combatir el colapso de la energía o de proporcionar electricidad a aquellos que aún no han tenido acceso a ella. La repetición constante de esta información errónea ha ganado una amplia aceptación en Brasil (Fearnside, 2019, p. 56). Sin embargo, el verdadero propósito de la energía generada por las hidroeléctricas que se pretenden construir nunca se discute, y eso tiene especial relevancia. Solo después de conocer el propósito se debería debatir sobre los impactos de estas obras. Pero no ocurre así.

Los proyectos de hidroeléctricas siempre ganan las disputas, y ello se debe a que el discurso de que el país "necesita" más energía se ha convertido en algo de sentido común. Además, en contraste con esto, solo se presentan opciones que, por regla general, provienen de una fuente no renovable (combustible fósil). Una vez que se impone como cierta la necesidad de generar más y más energía como una forma de alcanzar el desarrollo, se impide un debate más profundo sobre el propósito de la energía que se va a generar, y este punto es crucial en el debate sobre el sector en Brasil.

Buena parte de la energía generada por las centrales hidroeléctricas brasileñas, desde Tucuruí hasta Belo Monte, está destinada a producir alúmina y aluminio para la exportación (Pinto, 2012, pp. 778-782). Esto representa una de las peores opciones posibles en términos de generación de empleo en Brasil, ya que "el procesamiento de aluminio genera 1,46 empleos por gigavatio/hora de electricidad consumida". La primera 
medida para pensar de manera diferente sobre el escenario hidroeléctrico brasileño sería dejar de exportar aluminio y productos electro intensivos (Fearnside, 2019, p. 57).

En la primera década del siglo XIX, la industria brasileña era responsable del consumo del $50 \%$ de la energía generada en el país, la mitad de la cual se dirigía a solo seis sectores, a saber: metales no ferrosos (aluminio), metalurgia, acero (acero), papel y celulosa, cemento y petroquímicos. Al menos el $8 \%$ del consumo se "exportó incorporando electricidad a productos primarios como el aluminio, el acero y la celulosa, sectores con bajo valor añadido, que no incluyen mano de obra" (Brasil, 2003).

La colonialidad del poder (Quijano, 2009) también se revela aquí, porque los precios de estos productos exportados son determinados por los consumidores que están en los llamados países desarrollados, que también son los que evitan establecer tales industrias en sus países. Esta realidad incorpora a Brasil, en esta perspectiva de globalización, como productor de bienes con bajo valor añadido y alto consumo de energía. Los costos sociales, humanos y ambientales son todos para Brasil/Amazonia, y buena parte de los beneficios para los consumidores de otros países (Brasil, 2003).

Además de infravalorar los costos ambientales y sociales, el pensamiento tradicional y los planes gubernamentales no tienen en cuenta el potencial de mejorar la eficiencia del sector energético como forma de atender a las necesidades. La eficiencia es el recurso energético más barato y seguro. El uso eficiente y el ahorro de energía cuestan mucho menos que la generación o su compra, no provoca, además, contaminación ni impactos socioambientales, y puede ser, aún, responsable de apoyar los multiplicadores económicos y el empleo local (Brasil, 2003) ${ }^{11}$.

Sobre todo, la eficiencia proporciona, como mayor ventaja, el estímulo macroeconómico que proviene de la reubicación del capital, que puede alcanzar escalas multimillonarias en pocos años. Esto se debe a que las tecnologías que se pueden desarrollar localmente, como lámparas fluorescentes compactas o marcos de ventanas que bloquean el calor/frío, pueden requerir una inversión de capital hasta mil veces menor por kilovatio, mientras se mantiene la misma luz y comodidad para los usuarios.

Otro ejemplo para buscar la eficiencia energética en Brasil se refiere a una cuestión básica del día a día, el calentamiento del agua. Del 5 al 10\% de todo el consumo de electricidad en Brasil se destina a calentar agua, especialmente mediante el uso de duchas eléctricas. Brasil es uno de los pocos países del mundo que usa ducha eléctrica, y entre ellos es el que más la utiliza. Tales porcentajes exceden la capacidad de generación de energía de las mayores hidroeléctricas brasileñas (Brasil, 2003) ${ }^{12}$.

Mientras que el consumidor invierte un promedio de 30 reales para comprar una ducha eléctrica, el país invierte entre 2 y 3 mil reales para instalar la capacidad de generar la electricidad que necesita esa ducha eléctrica, además de todos los costos ambientales, sociales y humanos de estas fuentes de generación de energía. Otros medios, como el calentamiento solar, como prioridad, y el gas como alternativa, reorganizarían el consumo de energía para el calentamiento del agua (Fearnside, 2019, p. 57).

Con esta reestructuración de la economía y la eficiencia energética, se podría transformar "el sector energético de un agujero negro a una fuente neta de capital para financiar otras necesidades de desarrollo". Es posible emular resultados de mercado eficientes para que la solución de menor costo para el cliente sea también la más rentable para el proveedor (Brasil, 2003). Brasil es, de lejos, uno de los países más afortunados del mundo, porque tiene amplias alternativas a las plantas hidroeléctricas, los combustibles fósiles y la energía nuclear para satisfacer sus necesidades de electricidad. Brasil tiene "ricas reservas de megavatios inexploradas" (Brasil, 2003).

La aplicación del "principio de proporcionar servicios de energía a un costo mínimo y usar las herramientas de planificación integrada de recursos para alinear las recompensas empresariales e institucionales con los objetivos sociales a nivel nacional", podría dirigir al país hacia la superación de este paradigma en el sector y dar a los brasileños la "sociedad justa y próspera" que desea (Brasil, 2003). 
Los pasos hacia esta mejor eficiencia energética, en el sentido de renunciar a este patrón colonial de exportaciones con uso intensivo de energía eléctrica, la promoción e incentivos para una cultura de uso adecuado de la electricidad, y la búsqueda "para aprovechar los recursos solares y eólicos, está totalmente ausente de los planes del gobierno o recibe solo una consideración simbólica”. Esto se refleja en los planes de desarrollo más recientes, a ejemplo de los vetos presentados por la presidenta Dilma Rousseff, en enero de 2016, a las alternativas no hidroeléctricas para la producción de electricidad a gran escala en Brasil (Brasil, 2016).

\section{Conclusión}

No hay forma de justificar el progreso y el desarrollo con la imposición de culturas y pueblos, ya sea por prácticas genocidas, como ocurrió durante mucho tiempo en la Amazonia, o por lo que categorizamos como etnocidio, la faceta más perversa e invisible de los proyectos hidroeléctricos implementados en la Amazonia. No existe, por lo tanto, desde un punto de vista político-filosófico, ni histórico ni, claramente, legal, desde la Constitución de 1988, justificación para estos megaproyectos hidroeléctricos en la Amazonia que representan un daño a la sostenibilidad de sus pueblos y comunidades tradicionales.

El problema de investigación queda así contestado y la hipótesis confirmada, ya que ha sido posible percibir que las narrativas y discursos del gobierno que afirman que las fuentes hídricas de generación de energía eléctrica son limpias y sostenibles solo suponen una estrategia para que tales proyectos puedan ser aprobados y aceptados en la Amazonia. Así, el sector energético brasileño ha podido ser orientado hacia la Amazonia sin tener en cuenta todos los costos humanos y ambientales.

Habría opciones más viables para equilibrar y estabilizar el sector energético brasileño que no implican tales impactos socioambientales en la Amazonia, sin embargo, se insiste en mantener un modelo que solo realiza el análisis de los costos-beneficios a partir de una visión economicista, sin tener en cuenta los costos humanos y ambientales en esa ecuación. 


\section{NOTAS}

${ }^{1}$ Art. $2^{\circ}$. Compete ao Comitê: a) supervisionar os estudos visando a investigação das possibilidades de aproveitamento hidrelétrico para o suprimento de sistemas elétricos já existentes ou que venham a ser implantados em áreas prioritárias e polos de desenvolvimento criados na Amazônia pelo Governo Federal. b) Preparar, dentro das disponibilidades financeiras, o orçamento e aprovar o cronograma trimestral das despesas a serem efetuadas, indicando as fontes supridoras dos recursos; c) Aprovar e autorizar despesas; d) Deliberar sobre as soluções técnicas apresentadas pelos consultores, recomendando novos estudos, se julgar conveniente; e) Propor ao Ministro de Estado as providências que considerar necessárias à execução de seus serviços; f) Apresentar às entidades financiadoras, relatórios comprovações de despesas, e outros documentos.

2 Eletronorte inicialmente cubría los estados de Amazonas, Pará, Acre, Mato Grosso y Goiás, así como los territorios de Amapá, Roraima y Rondônia. Posteriormente se expandió, incluyendo Maranhão y el resto del estado de Mato Grosso.

2 Represas: 1. São Gabriel (2.000 MW); 2. Santa IsabelUaupés/Negro: (2.000 MW); 3. Caracaraí-Mucajaí (1.000 MW); 4. Maracá (500 MW); 5. Surumu (100 MW); 6. Bacarão (200 MW); 7. Santo Antônio [Cotingo] (200 MW); 8. Endimari (200 MW); 9. Madeira/Caripiana (3800 MW); 10. Samuel (200 MW); 11. Tabajara-JP-3 (400 MW); 12. Jaru-JP-16 (300 MW); 13. Ji-Paraná-JP-28 (100 MW); 14. Preto RV-6 (300 MW); 15. Muiraquitã RV-27 (200 MW); 16. Roosevelt RV-38 (100 MW); 17. Vila do Carmo AN-26 (700 MW); 18. Jacaretinga AN-18 (200 MW); 19. Aripuanã AN-26 (300 MW); 20. Umiris SR-6 (100 $M W) ; 21$. Itaituba (13.000 MW) 22. Barra São Manuel (6.000 MW); 23. Santo Augusto (2.000 MW); 24. Barra do Madeira [Juruena] (1000 MW); 25. Barra do Apiacás (2000 MW); 26. Talama [Novo Horizonte] (1.000 MW); 27. Curuá-Una (100 MW); 28. Belo Monte [Cararaô] (8.400 MW) 29. Babaquara (6.300 MW); 30. Ipixuna (2.300 MW); 31. Kokraimoro (1.900 MW); 32. Jarina (600 MW); 33. Iriri (900 MW); 34. Balbina (250 MW); 35. Fumaça (100 $\mathrm{MW}) ; 36$. Onça (300 MW); 37. Katuema (300 MW); 38. Nhamundá/Mapuera (200 MW); 39. Cachoeira Porteira (1.400 MW); 40. Tajá (300 MW); 41. María José (200 MW); 42. Treze Quedas (200 MW); 43. Carona (300 MW); 44. Carapanã (600 MW); 150 Mel (500 MW); 46. Armazém (400 MW); 47. Paciência (300 MW); 48. Curuá (100 MW); 49. Maecuru (100 MW); 50. Paru III (200 MW); 51. Paru II (200 MW); 52. Paru I (100 MW); 53. Jari IV (300 MW); 54. Jari III (500 MW); 55. Jari II (200 MW); 56. Jari I (100 MW); 57. F. Gomes (100 MW); 58. Paredão (200 MW); 59. Caldeirão (200 MW); 60. Arrependido (200 MW); 61. Santo Antônio [Araguari] (100 MW); 62. Tucuruí (6.600 MW); 63. Marabá (3.900 MW); 64. Santo Antônio [Tocantins] (1.400 MW); 65. Carolina (1.200 MW); 66. Lajeado (800 MW); 67. Ipueiras (500 MW); 68. São Félix (1.200 MW); 69. Sono II (200 MW); 70. Sono I (100 MW); 71. Balsas I (100 MW); 72. Itacaiúnas II (200 MW); 73. Itacaiúnas I (100 MW); 74. Santa Isabel (Araguaia) (2200 MW); 75. Barra do Caiapó (200 MW); 76. Torixoréu
(200 MW); 77. Barra do Peixe (300 MW); 78. Couto de Magalhães (200 MW); 79. Noidori (100 W).

4 Véase "3.1.2. O aproveitamento do potencial hidrelétrico da Amazônia", "5. Expansão ao longo prazo do sistema elétrico", e "6. Expansão a médio prazo do sistema elétrico".

${ }^{5}$ Los detalles sobre las obras en el sector energético se detallan en los Planes Decenales de Expansión de Energía, y no directamente en los Planes Plurianuales, que son planes más amplios que traen las pautas de inversión, pero sin detalles.

${ }^{6}$ Los Planes Decenales de Expansión de Energía son documentos informativos, emitidos por la Empresa de Investigación Energética (EPE) del Ministerio de Minas y Energía (MME), dirigidos a la sociedad, en los que se presentan las indicaciones y perspectivas del gobierno para la futura expansión del sector energético por períodos de diez años.

${ }^{7}$ Además de los trabajos/producciones científicas sobre el tema, la realización de esta tabla analizó los diversos planes decenales de expansión de energía. A través de las indicaciones de los planes, se realizó una investigación en internet para confirmar si las represas ya habían sido finalizadas y puestas en funcionamiento. Cabe señalar que solo se encontró una investigación en donde aparecen todas las represas existentes en la Amazonia Legal brasileña, pero solo hasta el año 2013 (Fearside, 2015 , p. 13), sin previsiones del período posterior. Por lo tanto, la tabla es el resultado de una serie de análisis y búsquedas, de manera que es posible que existan plantas hidroeléctricas que ya estén en funcionamiento y no se haya identificado en esta investigación.

${ }^{8}$ Las centrales hidrelétricas existentes son: 1) CoarcyNunes, 2) Curuá-Una, 3) Tucuruí, 4) Balbina, 5) Manso, 6) Samuel, 7) Lajeado, 8) Peixe Angelical, 9) São Salvador, 10) Dardonelos, 11) Rondon II, 12) Estreito, 13) Santo Antônio [Río Madeira], 14) Jirau, 15) Santo Antônio [Río Jari], 16) Teles Pires, 17) São Manoel, 18) Belo Monte, 19) Sinop, 20) Ferreira Gomes, 21) Colider, 22) Cachoeira Caldeirão, 23) Complexo Apaiacás. Algumas hidrelétricas planejadas: 1) Cachoeira Porteira, 2) Marabá, 3) São Luiz do Tapajós, 4) Babaquara/Altamira.

${ }^{9}$ Esta tabla se realizó a partir del análisis detallado de trabajos científicos (Fearside, 2015; 2019) y, principalmente, del análisis de los últimos 12 planes decenales de expansión energética, emitidos por el MME. Cabe señalar, sin embargo, que era necesaria una comparación entre los diferentes planes, esto se debe a que muchas indicaciones de plantas hidroeléctricas no ejecutadas se repiten en planes posteriores y otras, sin embargo, no se mencionan. Se decidió, por lo tanto, presentar las indicaciones de proyectos hidroeléctricos de manera decreciente, es decir, todas las plantas hidroeléctricas previstas en los planes más recientes se incluyeron en la tabla, posteriormente fueron incluidas las previstas en los planes más antiguos que 
no tuvieron secuencia en el más recientes, como forma de poder enumerar todas las plantas hidroeléctricas ya discutidas para la Amazonia en los diversos planes para la expansión de la energía. Debido a la complejidad de los planes decenales, es posible que alguna indicación no esté en la tabla.

10 Estudios exigidos por la ley brasileña para aquellos proyectos que utilizarán recursos ambientales y que tienen potencial de contaminación o degradación (Brasil, 1990).

11 "Substituindo esses chuveiros por aquecedores de água solares, a gás butano ou a gás natural mais eficientes, e instalar um fusível permanente que evite a reinstalação do chuveiro elétrico economizaria dinheiro e liberaria até pelo menos $10 \%$ da capacidade de geração nacional" (Brasil, 2003).

12 "A eficiência em termos de energia aumenta o emprego local ao criar e instalar tecnologias mais inteligentes, em vez de comprar energia de longe. A eficiência também pode beneficiar desproporcionadamente os pobres, para quem a energia absorve uma maior fração da renda disponível do que para os mais bem aquinhoados pela fortuna. Noventa por cento da iluminação residencial e metade da comercial no Brasil ainda é feita com lâmpadas incandescentes, principalmente por clientes que não podem pagar as lâmpadas eficientes, que são mais caras. Um dos principais serviços públicos dos Estados Unidos da América achou que valia a pena dar mais de um milhão de lâmpadas fluorescentes compactas de eficiência quintuplicada simplesmente para economizar em suas usinas de eletricidade existentes - sem falar no fato de evitar investimentos em novas usinas. A tecnologia eficiente em termos de energia pode também favorecer a consecução de objetivos sociais. Uma experiência em Curitiba mostrou que instalar 'prateleiras de luz' baratas nas janelas de salas de aula economizava $75 \%$ de uso de eletricidade, o que permitia à escola comprar mais livros. Também, os estudantes aprendem até $26 \%$ mais rápido em salas de aula bem iluminadas com a luz do dia" (Brasil, 2003). 


\section{REFERÊNCIAS BIBLIOGRÁFICAS}

A eficiência é o maior, mais barato e mais seguro recurso energético do Brasil. (2003, 11 ago.). Portal do Ministério do Meio Ambiente. Recuperado de [https://www.mma. gov.br/informma/item/1425-a-eficiencia-e-o-maiormais-barato-e-mais-seguro-recurso-energetico-dobrasil.html]. Consultado [27-03-2020].

Baitelo, R. et al. (2013). [R]evolução energética: a caminho do desenvolvimento. São Paulo: Greenpeace Brasil. Recuperado de [http://www.greenpeace.org/brasil/pt/ Documentos/Revolucao-Energetica-/]. Consultado [2004-2020].

Batista, I. M. S., \& Miranda, L. M. (2019) Os "Hidronegócios" nos rios da Amazônia. Revista Brasileira de História, 39(81), 117-139.

Bermann, C. (2002) Energia no Brasil, para que? Para quem?: crise e alternativa para um país sustentável. Editora Livraria da Física.

Braga, D. C. (2019). Energia nuclear entre o paradigma da sustentabilidade e a transnacionalidade: possibilidades jurídicas para sua expansão na matriz elétrica brasileira no terceiro milênio. Tesis de doctorado, Universidade do Vale do Itajaí, Itajaí, Santa Catarina, Brasil.

Carvalho, G. (2001). Histórico e Impacto das Políticas Públicas na Amazônia. In A. C. Barros (Org.). Sustentabilidade e Democracia para as políticas públicas na Amazônia. Río de Janeiro: Projeto Brasil Sustentável e Democrático, FASR/IPAM.

Chaves, K. A. (2019). Colonialidade, exceção-espoliação e etnocídio: o caso dos atingidos à jusante da barragem de Belo Monte. Terra Livre, 2(51), 120-152.

Constituição dos Estados Unidos do Brasil (1946). Río de Janeiro: Assembleia Constituinte. Recuperado de [http://www.planalto.gov.br/ccivil_03/constituicao/ constituicao46.htm]. Consultado [24-03-2020].

Corrêa, C. G. et al. (1994). O processo de ocupação humana na Amazônia: considerações e perspectivas. Bol. Museu Paraense Emilio Goeldi, Sér. Antropol. 9(1). Recuperado de [https://repositorio.museu-goeldi.br/ handle/mgoeldi/814]. Consultado [11-03-2020].

Decreto $n^{\circ}$ 96.652, de 6 de setembro de 1988. (1988). Aprova o Plano Nacional de Energia Elétrica 1987/2010 Plano 2010 -, fixa diretrizes e normas para concessão ou autorização de centrais geradoras de energia elétrica no País e dá outras providências. Diário Oficial da União de 08/09/1988, pp. 17-217.

Decreto $n^{\circ}$ 63.952/1968. (1969). Cria no Ministério das Minas e Energia o Comitê Coordenador dos Estudos Energéticos da Amazônia. Diário Oficial da União, Seção 1, de 6 de jan. de 1969, p. 65. Recuperado de [https:// www2.camara.leg.br/legin/fed/decret/1960-1969/ decreto-63952-31-dezembro-1968-405481publicacaooriginal-1-pe.html]. Consultado [24-03-2020].
Decreto $n^{\circ} 72.548 / 1973$. (1973). Concede autorização para funcionar como empresa de energia elétrica à Centrais Elétricas do Norte do Brasil S. A. ELETRONORTE. Diário Oficial da União, em 31/7/1973, Seção 1, pp. 7-481. Recuperado de [https://www2. camara.leg.br/legin/fed/decret/1970-1979/decreto72548-30-julho-1973-378831-publicacaooriginal-1-pe. html]. Consultado [24-03-2020].

Decreto $n^{\circ}$ 99.274/1990. (1990). Regulamenta a Lei $n^{\circ}$ 6.902, de 27 de abril de 1981, e a Lei ${ }^{\circ} 6.938$, de 31 de agosto de 1981, que dispõem, respectivamente sobre a criação de Estações Ecológicas e Áreas de Proteção Ambiental e sobre a Política Nacional do Meio Ambiente, e dá outras providências. Diário Oficial da União de 07/06/1990, p. 10.887. Recuperado de [http://www. planalto.gov.br/ccivil_03/decreto/Antigos/D99274.htm]. Consultado [30-03-2020].

Fearnside, P. M. (2006). Dams in the Amazon: Belo Monte and Brazil's Hydroelectric Development of the Xingu River Basin. Environmental Management, 38(16). Recuperado de [https://link.springer.com/article/10.1007/s00267005-0113-6]. Consultado [26-03-2020].

Fearnside, P. M. (2015). Hidrelétricas na Amazônia: impactos ambientais e sociais na tomada de decisões sobre grandes obras (Vol. 2). Manaos: INPA.

Fearnside, P. M. (2019a). Hidrelétricas na Amazônia: impactos ambientais e sociais na tomada de decisões sobre grandes obras (Vol. 3). Manaos: INPA.

Fearnside, P. M. (2019b). Represas hidroeléctricas en la Amazonia brasileña: impactos ambientales y sociales. Revista de Estudios Brasileños, 6(11), 123-138.

Fearnside, P. M., \& Laurance, W. F. (2012). Infraestrutura na Amazônia: as lições dos planos plurianuais. Caderno $\mathrm{CRH}$, 25(64), 87-98.

Garcia, M. F. (2004). Ocupação do território e impactos ambientais: o papel dos grandes projetos de eletrificação da Amazônia. II Encontro da ANNPAS (on-line). São Paulo: ANNPAS. Recuperado de [http://www.anppas. org.br/encontro_anual/encontro2/GT/GT06/m\%E1rcia_ feitosa.pdf]. Consultado [24-03-2020].

Il Plano de desenvolvimento da Amazônia. (1975-79). (1976). Superintendência da Amazônia. Belém: Divisão de Documentação.

Junk, W. J., \& Mello, J. A. S. (1987). Impactos ecológicos das represas hidrelétricas na bacia amazônica brasileira. In G. Kohlhepp, \& A. Schrader. Homem e natureza na Amazônia: Simpósio Internacional e Interdisciplinar, Associação Alemã de Pesquisas sobre a América Latina (ADLAF).

Lei $n^{\circ}$ 1.806/1953. (1953) Dispõe sobre o Plano de Valorização Econômica da Amazônia, cria a superintendência da sua execução e dá outras providências. Diário Oficial da União de 7/1/1953, Seção 
1, p. 276. Recuperado de [http://www.planalto.gov.br/ ccivil_03/leis/1950-1969/L1806.htm]. Consultado [2403-2020].

Lei $n^{\circ}$ 10.933/2003. (2003). Dispõe sobre o Plano Plurianual para o período 2004/2007. Diário Oficial da União de 12/08/2004, p. 1. Recuperado de [http:// www.planalto.gov.br/ccivil_03/_Ato2004-2006/2004/Lei/ L10.933.htm]. Consultado [24-03-2020].

Lei $n^{\circ}$ 12.593/2012. (2012). Institui o Plano Plurianual da União para o período de 2012 a 2015. Diario Oficial da União de 19/01/2012, p. 1. Recuperado de [http:// www.planalto.gov.br/ccivil_03/_ato2011-2014/2012/Lei/ L12593.htm]. [Consultado 24-03-2020].

Lei $n^{\circ}$ 3.890-A/1961. (1961). Autoriza a União a constituir a empresa Centrais Elétricas Brasileiras S. A. - ELETROBRÁS, e dá outras providências. Diário de 25 de abril de 1961. Recuperado de [https://www2.camara. leg.br/legin/fed/lei/1960-1969/lei-3890-a-25-abril-1961353665-normaatualizada-pl.html]. Consultado [24-032020].

Lei $n^{\circ}$ 5.824/1972. (1972). Dispõe sobre empréstimo compulsório, em favor da Centrais Elétricas Brasileiras S.A. -Eletrobras. Diário Oficial da União de 17/11/1972. Recuperado de [http://www.planalto.gov.br/ccivil_03/ leis/L5824.htm]. Consultado [24-03-2020].

Lei $n^{\circ}$ 9.276/1996. (1996). Dispõe sobre o Plano Plurianual para o período de 1996/1999 e dá outras providências. Diário Oficial da União de 10/05/1996, p. 7947. Recuperado de [http://www.planalto.gov.br/ ccivil_03/LEIS/L9276.htm]. Consultado [24-03-2020].

Lei $n^{\circ}$ 9.989/2000. (2000). Dispõe sobre o Plano Plurianual para o período de 2000/2003. Diário Oficial da União de 24/07/2000, p. 1. Recuperado de [http://www. planalto.gov.br/ccivil_03/leis/L9989.htm]. Consultado [24-03-2020].

Lei $n^{\circ}$ 11.653/2008. (2008). Dispõe sobre o Plano Plurianual para o período 2008/2011. Diário Oficial da União de 08/04/2008, p. 5. Recuperado de [http:// www.planalto.gov.br/ccivil_03/_ato2007-2010/2008/lei/ I11653.htm]. Consultado [24-03-2020].

Mensagem $n^{\circ}$ 16/2016. (2016). Comunico a Vossa Excelência que, nos termos do $\S 1^{\circ}$ do art. 66 da Constituição, decidi vetar parcialmente, por inconstitucionalidade e contrariedade ao interesse público, o Projeto de Lei $n^{\circ} 6$, de $2015-\mathrm{CN}$, que "Institui o Plano Plurianual da União para o período de 2016-2019". Recuperado de [http://www.planalto.gov.br/ccivil_03/_ ato2015-2018/2016/Msg/VEP-16.htm]. Consultado [1305-2020].

Moreira, P. F. (2012). Setor Elétrico Brasileiro e a Sustentabilidade no Século 21: Oportunidades e Desafios. 2a ed. Brasilia: Rios Internacionais. Recuperado de [http://www.internationalrivers.org/ node/7525]. Consultado [20-04-2020].
Pasold, C. L. (2015). Metodologia da pesquisa jurídica: teoria e prática (13 ${ }^{\mathrm{a}}$ Ed.). Florianópolis: Conceito Editorial.

Pinto, L. F. (2012). De Tucuruí a Belo Monte: a história avança mesmo? Boletim do Museu Paraense Emílio Goeldi. Ciências Humanas, 7(3), 777-782.

Plano Decenal de Expansão de Energia 2015. (2006). Brasilia: MME/EPE, 2006. Recuperado de [http://www. epe.gov.br/pt/publicacoes-dados-abertos/publicacoes/ plano-decenal-de-expansao-de-energia-pde]. Consultado [26-03-2020].

Plano Decenal de Expansão de Energia 2016. (2007). Brasilia: MME/EPE, 2007. Recuperado de [http://www. epe.gov.br/pt/publicacoes-dados-abertos/publicacoes/ plano-decenal-de-expansao-de-energia-pde]. Consultado [26-03-2020].

Plano Decenal de Expansão de Energia 2017. (2008). Brasilia: MME/EPE, 2008. Recuperado de [http://www. epe.gov.br/pt/publicacoes-dados-abertos/publicacoes/ plano-decenal-de-expansao-de-energia-pde]. Consultado [26-03-2020].

Plano Decenal de Expansão de Energia 2020. (2011). Brasilia: MME/EPE, 2011. Recuperado de [http://www. epe.gov.br/pt/publicacoes-dados-abertos/publicacoes/ plano-decenal-de-expansao-de-energia-pde]. Consultado [26-03-2020].

Plano Decenal de Expansão de Energia 2022. (2013). Brasilia: MME/EPE, 2013. Recuperado de [http://www. epe.gov.br/pt/publicacoes-dados-abertos/publicacoes/ plano-decenal-de-expansao-de-energia-pde]. Consultado [26-03-2020].

Plano Decenal de Expansão de Energia 2023. (2014). Brasilia: MME/EPE, 2014. Recuperado de [http://www. epe.gov.br/pt/publicacoes-dados-abertos/publicacoes/ plano-decenal-de-expansao-de-energia-pde]. Consultado [26-03-2020].

Plano Decenal de Expansão de Energia 2024. (2015). Brasilia: MME/EPE, 2015. Recuperado de [http://www. epe.gov.br/pt/publicacoes-dados-abertos/publicacoes/ plano-decenal-de-expansao-de-energia-pde]. Consultado [26-03-2020].

Plano Decenal de Expansão de Energia 2029. (2020). Brasilia: MME/EPE, 2020. Recuperado de [http://www. epe.gov.br/pt/publicacoes-dados-abertos/publicacoes/ plano-decenal-de-expansao-de-energia-pde]. Consultado [26-03-2020].

Plano Nacional de Energia Elétrica 1987/2010. (1987). Ministério das Minase Energia-MME. Relatório Executivo. Rio de Janeiro: MME, 1987. Recuperado de [http://www. planalto.gov.br/ccivil_03/decreto/1980-1989/anexo/ and96652-88.pdf]. Consultado [24-03-2020].

Quijano, A. (2009). Colonialidade do Poder e Classificação Social. In B. S. Santos, \& M. P. Meneses. Epistemologias do Sul. Coimbra: Edições Almedina. SA. 